\title{
Resonantly driven coherent oscillations in a solid-state quantum emitter
}

\author{
E. B. Flagg ${ }^{1 \star}$, A. Muller ${ }^{2}$, J. W. Robertson ${ }^{1}$, S. Founta ${ }^{1}$, D. G. Deppe ${ }^{3}$, M. Xiao ${ }^{4}$, W. Ma ${ }^{4}$, G. J. Salamo ${ }^{4}$ \\ and C. K. Shih ${ }^{1 \star}$
}

Single-quantum emitters emit only one photon at a time ${ }^{1,2}$, but the properties of the photon depend on how the emitter is excited ${ }^{3}$. Incoherent excitation is simple and broadly used with solid-state emitters such as quantum dots, but does not allow direct manipulation of the quantum state. Coherent, resonant excitation on the other hand is used in pump-probe techniques to examine the quantum state of the emitter ${ }^{4}$, but does not permit collection of the single-photon emission. Coherent control with simultaneous generation of photons has been an elusive goal in solid-state approaches, where, because of strong laser scattering at the detection wavelength, measurement of resonant emission has been limited to cross-polarized detection ${ }^{5}$ or Stokes-shift techniques ${ }^{6,7}$. Here we demonstrate that a semiconductor quantum dot in a microcavity can be resonantly driven and its single-photon emission extracted background free. Under strong continuouswave excitation, the dot undergoes several Rabi oscillations before emitting, which are visible as oscillations in the second-order correlation function. The quantum-dot states are therefore 'dressed', resulting in a Mollow-triplet emission spectrum. Such coherent control will be necessary for future high-efficiency sources of indistinguishable single photons $\mathrm{s}^{3,8}$, which can be used for quantum key distribution ${ }^{9}$ or through post-selection $^{10}$ to generate entangled photon pairs ${ }^{11,12}$.

Semiconductor quantum dots (QDs) are collections of thousands of atoms, whose electronic degrees of freedom are discretized, similar to those of an atom, owing to confinement of electrons and holes. Therefore, a number of quantum optical and quantum electrodynamic phenomena familiar from atomic physics that have wide implications in quantum information science could in principle be realized in monolithic, scalable solid-state systems. Coherent control of the exciton state is required for numerous applications, and has been demonstrated in many previous works showing Rabi splitting ${ }^{13}$, Rabi oscillations ${ }^{14-16}$, Autler-Townes splitting and Mollow absorption ${ }^{4}$, although these do not resonantly detect the photon emission. In addition to pump-probe-type approaches, non-resonant exciton generation through excited QD states or a continuum has previously been used for discriminating the QD emission from the scattered laser background, but at the cost of sacrificing coherence. Although simultaneous resonant excitation and emission is routinely done in single atoms or ions ${ }^{17,18}$, it has been difficult in the solid state because scattered laser light overwhelms the weak single-photon emission. Recent demonstration of resonance fluorescence from single QDs in a microcavity ${ }^{19}$ and single molecules under a solid immersion lens ${ }^{5}$, however, will enable explorations of coherent control and emission in the solid state. Of these two methods, the microcavity approach used here achieves orders-of-magnitudebetter background suppression and enables measurement of the Mollow emission spectrum with virtually no contamination from the excitation laser.

First described in atoms by Mollow in 1969 (ref. 20), resonance fluorescence is the emission of a two-level quantum system under resonant excitation. The two levels under consideration here are the crystal ground state, $|0\rangle$, and one of the fine-structure-split single-exciton states, $|1\rangle$. They are coupled by the electric-dipole interaction, and under strong continuous-wave (CW) driving fields the resulting energy levels are best described by the 'dressed-state' picture $^{21}$. Figure 1a shows the evolution from the 'bare' states to dressed states as the electric-field amplitude is increased. In the dressed-state picture there are four radiative transitions: two degenerate ones at the exciton ground-state energy, $\hbar \omega_{0}$, and two that are displaced by the Rabi energy, $\hbar \omega_{0} \pm \hbar \Omega$, where $\Omega=\kappa E / \hbar$, $\kappa$ is the QD dipole moment and $E$ is the electric-field amplitude. Together they form the Mollow-triplet emission, where the central peak has twice the intensity of the sidebands. As the dressed-state splitting is proportional to the electric field, the separation of the sidebands increases linearly with the square root of the laser power.

The first-order coherence as observed in the emission spectrum, however, is not sufficient to illustrate the quantum nature of the emitted photons. To do so, it is necessary to measure the secondorder correlation function, $g^{(2)}(\tau, t)=\langle I(t) I(t+\tau)\rangle /\langle I(t)\rangle^{2}$, where $I(t)$ is the emission intensity as a function of time. In CW excitation $g^{(2)}(\tau, t)$ is independent of the value of $t$ and can be written $g^{(2)}(\tau)$. The commonly known antibunching dip in $g^{(2)}(\tau)$ at $\tau=0$ is the signature of a single quantum emitter and has been observed in many solid-state systems ${ }^{1,2,8}$ under non-resonant excitation. When the quantum emitter is resonantly driven into the nonlinear regime, however, the situation is quite different. In the case of CW excitation, the system will undergo several Rabi oscillations before emitting, resulting in oscillations in $g^{(2)}(\tau)$. These oscillations are visible because the detection of one photon prepares the system in the ground state, $|0\rangle$, and the subsequent coherent evolution is seen in the probability of detecting a second photon a time $\tau$ later.

To investigate Mollow fluorescence and oscillatory $g^{(2)}(\tau)$, we resonantly excite a single quantum dot with a narrow-band $\mathrm{CW}$ laser using the waveguide mode of a planar microcavity, as explained in the Methods section. The microcavity greatly suppresses laser scattering because the excitation light is contained by total internal reflection. When the laser frequency is resonant with the excitonic ground state of a QD, the resonance fluorescence

${ }^{1}$ Department of Physics, The University of Texas at Austin, Austin, Texas 78712, USA, ${ }^{2}$ Joint Quantum Institute, NIST and University of Maryland, Gaithersburg, Maryland 20899, USA, ${ }^{3}$ College of Optics and Photonics (CREOL), University of Central Florida, Orlando, Florida 32816, USA, ${ }^{4}$ Department of Physics, University of Arkansas, Fayetteville, Arkansas 72701, USA. *e-mail: ebflagg@physics.utexas.edu; shih@physics.utexas.edu. 
a

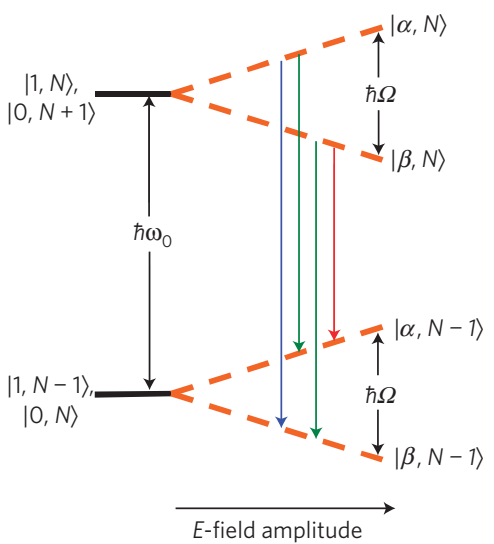

C

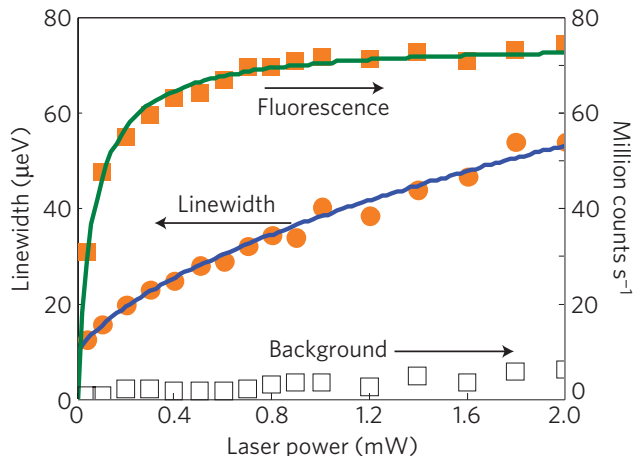

b

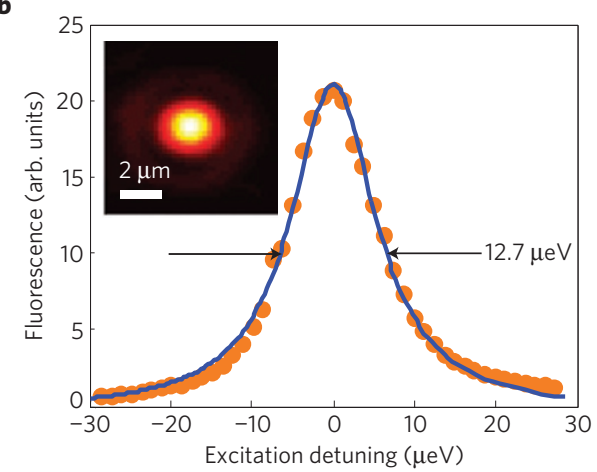

d

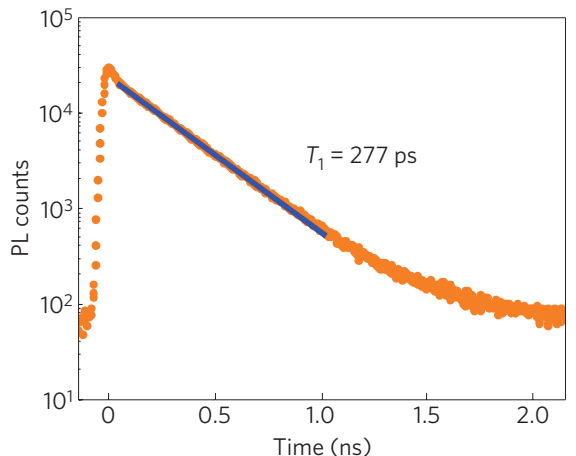

Figure 1 | Resonance fluorescence. a, Evolution of bare states to dressed states as the driving field is increased. $\mathbf{b}$, Total integrated fluorescence intensity of QD1 as a function of excitation detuning. The linewidth of this particular dot is $12.7 \mu \mathrm{eV}$ at low power. The inset shows a spatial image of the fluorescence; the diffraction-limited spot size is $\sim 2 \mu \mathrm{m}$ in diameter and a faint Airy ring can be seen around the central peak. c, Power broadening and saturation of QD1. The error bars are on the order of the size of the points and the low-intensity limit of the linewidth is $2 \hbar \Gamma=9.936 \pm 0.760 \mu \mathrm{eV}$, corresponding to a coherence time $T_{2}=132 \pm 10 \mathrm{ps}$. The background level is very low even at the highest intensities used. Below saturation the fluorescence intensity can be up to 100 times greater than the scattered background due to enhancement of the emission by cavity coupling and the orthogonality of the excitation and collection directions. d, Time-resolved emission intensity of QD1. The line is a fit with a decay time of $T_{1}=277 \pm 30 \mathrm{ps}$.

is observed as a bright peak in the spectrometer images, localized both spectrally and spatially. The inset of Fig. 1b shows a spatial image of the fluorescence. The laser polarization and propagation are in the sample plane. The fluorescence propagation is normal to the plane and its polarization is parallel to that of the laser, so no polarization discrimination was used (see Supplementary Information, Fig. S1 or ref. 19 for a schematic diagram of the excitation geometry). The QD population, and therefore fluorescence, are given by

$$
n_{\infty}=\frac{1}{2} \frac{\Omega^{2} T_{1} / T_{2}}{\Delta \omega^{2}+T_{2}^{-2}+\Omega^{2} T_{1} / T_{2}}
$$

where $\Delta \omega$ is the laser detuning from the QD transition, $T_{1}$ is the QD lifetime and $T_{2}$ is the dephasing time. The dependence of the fluorescence intensity on the detuning enabled us to record the resonant photoluminescence excitation spectrum shown in Fig. 1b. Examining equation (1) shows that the linewidth power broadens with increased excitation intensity, as shown in Fig. 1c. The fit is derived from equation (1) and reveals a zero-intensity limit of $9.94 \pm 0.76 \mu \mathrm{eV}$, corresponding to a dephasing time of $T_{2}=132 \pm 10$ ps. Also plotted in Fig. 1c are the fluorescence intensity, which saturates at high powers, and the residual laser scattering, which is only a small fraction of the fluorescence even at the highest power used. Time-resolved resonance fluorescence from QD1 (using 5 ps laser pulses) is shown in Fig. 1d and the extracted lifetime is $T_{1}=277 \pm 30 \mathrm{ps}$, which is somewhat short for self-assembled QDs. $T_{1}$ may be reduced by a Purcell effect due to the cavity ${ }^{22,23}$, though the spectral overlap of the dot and cavity cannot be tuned so the magnitude of the effect cannot be measured. Alternatively, QDs which show easily observable nonlinear phenomena will have large dipole moments and therefore shorter lifetimes.

Figure 2a shows the normalized correlation data from QD1 at varying powers. The oscillations at high powers are well resolved for about 1.5 periods on either side, and at the lowest power the dip at $\tau=0$ is $50 \%$. In order to compare the measurement directly with the model function, the effects of a small background and the Hanbury Brown-Twiss (HBT) instrument response function (IRF) were removed as described in the Methods section. The IRF, shown in Fig. 2c, is the time response of the detectors and was measured using a 5 ps pulsed Ti:sapphire laser. Although the central peak of the IRF is narrow, the long-time tail has a significant effect on the measurement. Figure $2 \mathrm{~b}$ shows the measured $g^{(2)}(\tau)$ normalized to the long-time value after subtracting the background and deconvolving the IRF. After this deconvolution, at $\tau=0$ the antibunching dip is nearly ideal for every power level. Moreover, the data are fitted very well by the theoretically predicted $g^{(2)}(\tau)$ function ${ }^{24}$, which we have extended to include pure dephasing in addition to radiative recombination:

$$
g^{(2)}(\tau)=1-\exp [-\eta|\tau|]\left\{\cos (\mu|t|)+\frac{\eta}{\mu} \sin (\mu|t|)\right\}
$$

where $\eta=\left(1 / T_{1}+1 / T_{2}\right) / 2$ and $\mu=\sqrt{\Omega^{2}+\left(1 / T_{1}-1 / T_{2}\right)^{2}}$. Significantly, a single set of $T_{1}, T_{2}$ values, directly obtained above 

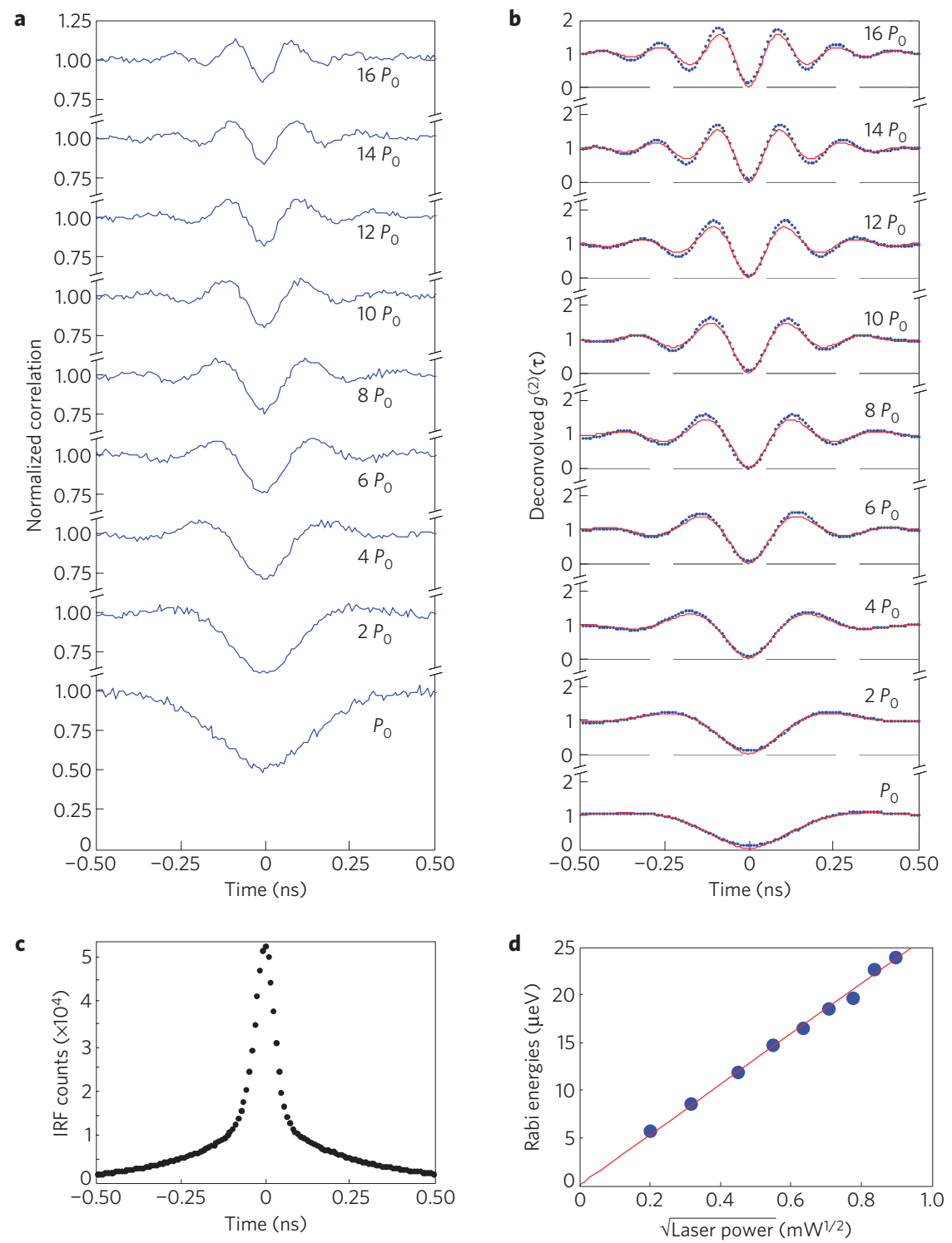

Figure 2 | Second-order correlations and Rabi energies. a, Raw second-order correlation data from QD1 at different excitation powers, normalized to the long-time value. $P_{0}=0.05 \mathrm{~mW}$. b. Second-order correlation data from QD1 after background subtraction and deconvolution of the IRF (see the Methods section for the deconvolution details). The lines are fits to equation (2) with $T_{1}=277 \mathrm{ps}$ and $T_{2}=132 \mathrm{ps}$. c) IRF of the HBT set-up for measuring $g^{(2)}(\tau)$. d, Rabi energies extracted from the fits in $\mathbf{b}$ plotted versus the square root of the laser power in the excitation fibre. The line is a linear fit to the data.

from time-resolved and linewidth measurements, fits the data at every excitation power level. Although the emission is not radiatively limited $\left(T_{2}<2 T_{1}\right)$, probably owing to spectral diffusion ${ }^{25}$ and/or pure dephasing, a constant value of $T_{2}$ indicates that there is no excitation-induced dephasing. Note that excitation-induced dephasing is different from the power broadening of Fig. 1c, which is an intrinsic phenomenon of resonant excitation predicted by the optical Bloch equations and occurs even with a constant $T_{2}$. Dephasing due to high intensities is a commonly observed phenomenon in solid-state systems ${ }^{13-16}$, but we and others ${ }^{26}$ find none using resonant excitation. The Rabi energies extracted from the fits to the correlation data are plotted in Fig. $2 \mathrm{~d}$ versus the square root of the laser power along with a linear fit that matches the data fairly well. Although coherently driven Rabi oscillations have been observed in the past ${ }^{14-16}$, those measurements were time-averaged. In contrast, $g^{(2)}(\tau)$ is a time-sensitive measurement that can show the Rabi oscillations as they occur, with the antibunching dip illustrating the quantum nature of the emission in a way that no previous measurement has. The conditional measurement of the
HBT set-up enables the first measurement of Rabi oscillations due to $\mathrm{CW}$ excitation. Indeed, Fig. $2 \mathrm{~b}$ and equation (2) have the same form as Torrey's transient solutions to the optical Bloch equations ${ }^{27}$.

The Mollow-triplet spectra at different excitation powers were obtained from a separate dot, QD2, and are plotted in Fig. 3. These measurements are background free because the central peak is much stronger than the laser scattering, as demonstrated by Fig. 1c. At low power the spectrum is a simple peak, which develops shoulders and finally sidebands, which move away from the central peak as the power is increased. The data are fitted by the function derived in refs 20,24 and extended to include pure dephasing, which on resonance is

$$
\begin{aligned}
S(\Delta v)= & \frac{n_{\infty}}{\pi}\left\{\frac{1}{2} \frac{1 / T_{2}}{(\Delta v)^{2}+1 / T_{2}^{2}}+\frac{n_{\infty}}{\Omega^{2}}\left(\frac{A \eta / 2-B(\Delta v-\mu) / 8 \mu}{(\Delta v-\mu)^{2}+\eta^{2}}\right.\right. \\
& \left.\left.+\frac{A \eta / 2-B(\Delta v+\mu) / 8 \mu}{(\Delta v+\mu)^{2}+\eta^{2}}\right)\right\}
\end{aligned}
$$




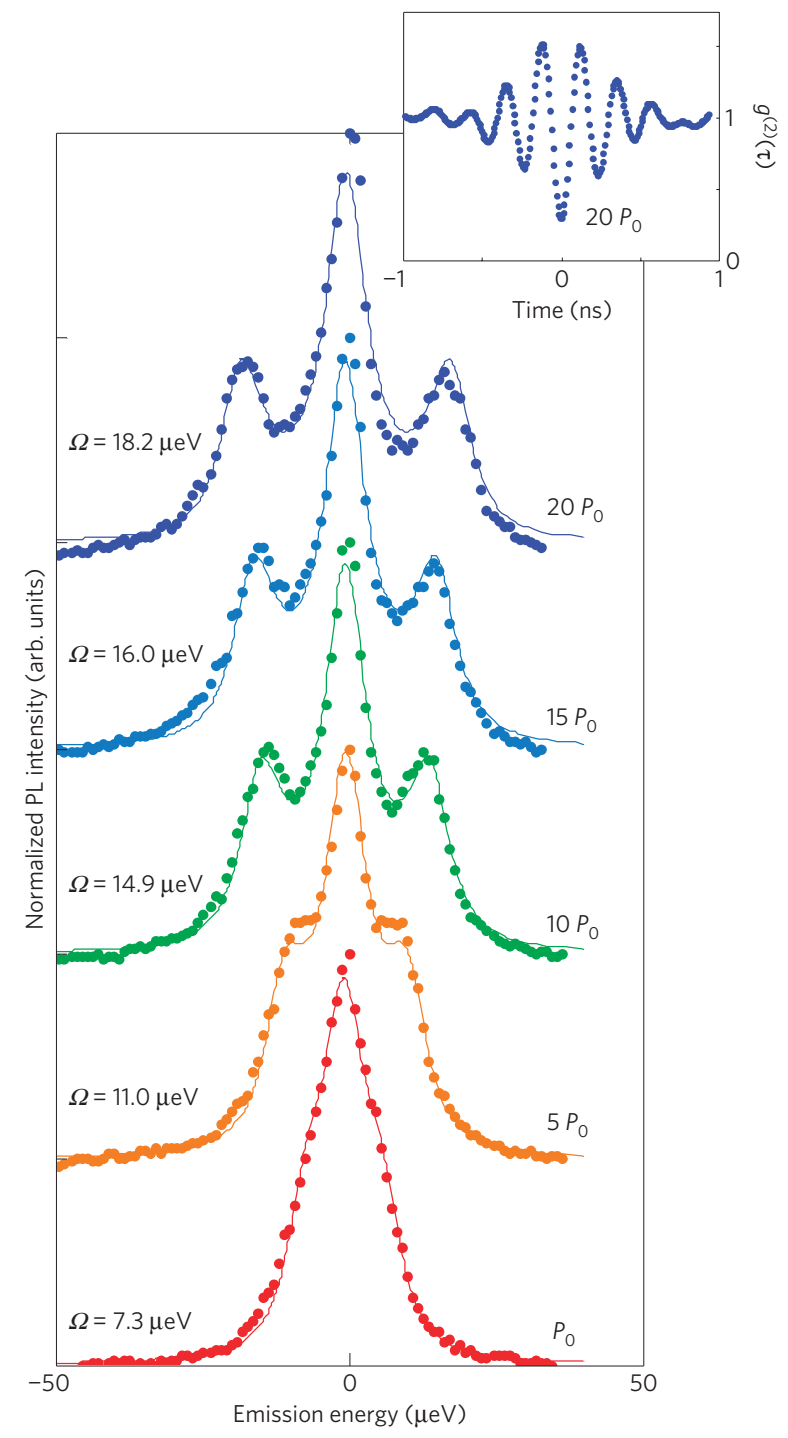

Figure 3 | Mollow triplet. Normalized emission spectra from QD2 at different excitation powers $\left(P_{0}=0.2 \mathrm{~mW}\right)$. Lines are fits to equation (3) with $T_{1}=149 \pm 13 \mathrm{ps}$ and $T_{2}=154 \pm 7 \mathrm{ps}$, and the Rabi energies are noted on the plot. The inset shows that QD2 also shows oscillatory $g^{(2)}(\tau)$, though the dip in the centre does not reach zero in this case. The full set of $g^{(2)}(\tau)$ data for QD2 is shown in Supplementary Information, Fig. S2.

where $n_{\infty}$ is the steady-state population from equation (1), $\Delta v$ is the detuning of the Fabry-Perot interferometer (FPI) from the resonant frequency and the constants $A$ and $B$ are

$$
\begin{gathered}
A=\Omega^{2}+\left(1 / T_{1}-1 / T_{2}\right) / T_{1} \\
B=2 \Omega^{2}\left(3 / T_{1}-1 / T_{2}\right)-2\left(1 / T_{1}-1 / T_{2}\right)^{2} / T_{1}
\end{gathered}
$$

Similar to the correlation data from QD1, a single pair of $T_{1}, T_{2}$ values suffices to fit the triplet data for every power level. From equation (3) it is clear that the width of the central peak is given by $2 / T_{2}$, and if excitation-induced dephasing were present $T_{2}$ would decrease and the peak would broaden at higher powers. As the peak width remains the same within the resolution of the detector for all powers, we conclude that there is no excitation-induced dephasing. For QD2, $T_{1}=149 \pm 13 \mathrm{ps}$ and $T_{2}=154 \pm 7 \mathrm{ps}$, and the extracted Rabi energies are labelled in the plots. Also acquired simultaneously for QD2 are the second-order correlation functions like those for
QD1. One such deconvolved trace is shown as an inset in Fig. 3 for the highest excitation power.

Mollow-triplet emission and an oscillatory second-order correlation function are signatures of strongly driven resonance fluorescence, which have been predicted but before now never observed in a semiconductor system. In addition to providing the first demonstration of these landmarks in a quantum dot, there are important implications in the broader quantum information sciences. Coherent manipulation combined with photon extraction will enable many applications where incoherent excitation is insufficient. The efficiency of a single-photon source may be increased by Purcell enhancement of the emission rate ${ }^{22,28}$, but in an incoherently excited source this results in a decrease of the indistinguishability ${ }^{3}$. This drawback, however, does not exist for coherent excitation such as resonance fluorescence ${ }^{3}$. Thus very low background resonant excitation similar to that demonstrated here could be used in a cavity with a high Purcell factor to create a high-efficiency single-photon source with indistinguishability greater than that possible with incoherent excitation.

\section{Methods}

The sample consisted of three layers of $\operatorname{In}_{0.35} \mathrm{Ga}_{0.65}$ As QDs separated by $17 \mathrm{~nm}$, grown between two distributed Bragg reflector (DBR) mirrors formed by alternating 1/4-wavelength layers of GaAs and AlAs with 18 periods on the bottom and 11 periods on the top. The separation between the DBRs was $1.16 \mu \mathrm{m}$ and the QD layers were $0.54 \mu \mathrm{m}$ from the bottom DBR. The main cavity mode was located near $914 \mathrm{~nm}$, with a full-width at half-maximum of about $5 \mathrm{~nm}$. More details can be found in ref. 29 .

The sample was maintained at low temperature $(10 \mathrm{~K})$ in a liquid-helium cryostat, and a polarization-maintaining single-mode optical fibre, mounted on a three-axis inertial walker, was brought close to the cleaved sample edge. The fibre was oriented so the polarization axes were aligned with the sample plane and sample normal. Through the fibre, the excitation laser was coupled into the waveguide mode of the cavity. Some fraction of the QDs resonantly coupled to a vertical mode of the cavity and the emission was collected by a high-numerical-aperture microscope objective. The microcavity greatly suppressed laser scattering because the laser was contained by total internal reflection between the DBR mirrors.

The emitted photons were directed either into an HBT set-up to measure $g^{(2)}(\tau)$, or into an imaging spectrometer with a two-dimensional CCD (charge-coupled device) array to measure the emission intensity and wavelength. Few spectrometers have the resolution necessary to distinguish the Mollow triplet; therefore, a scanning FPI was placed in front of the spectrometer and the transmitted intensity was recorded on the CCD as the FPI was scanned over the emission wavelength. This combination gave rise to an effective energy resolution of $1.2 \mu \mathrm{eV}$. The HBT set-up comprised a beamsplitter, two single-photon detection modules from Micro Photon Devices and a PicoHarp 300 time-correlated single-photon counting system.

CW excitation was provided by a tunable Ti:sapphire ring laser; a $5 \mathrm{ps}$ pulsed Ti:sapphire laser was used to measure the IRF of the HBT set-up. The exact laser intensity delivered to the $\mathrm{QD}$ varies between dots because the coupling efficiency of the light into the waveguide and the slight divergence within the waveguide are unknown. The powers noted in the figures are the amounts coupled into the excitation fibre. The FPI used to measure the Mollow triplet had a free spectral range of $30 \mathrm{GHz}(124 \mu \mathrm{eV})$ and a finesse of $\sim 100$, resulting in a transmission bandwidth of $0.3 \mathrm{GHz}(1.24 \mu \mathrm{eV})$.

The background in $g^{(2)}(\tau)$ was measured by simply tuning the laser off-resonance and recording the count rate. Deconvolution was accomplished in the Fourier domain by forming a modified Wiener filter out of the measured IRF, multiplying the data with the filter, then transforming back into the time domain $^{30}$. The modified Wiener filter is the theoretically optimum linear filter for data affected by additive Gaussian noise and an IRF. The filter function is given by $Y(\omega)=\left(\tau(\omega) \phi_{\mathrm{o}}(\omega) /\left(|\tau(\omega)|^{2} \phi_{\mathrm{o}}(\omega)+\phi_{\mathrm{n}}(\omega)\right)\right)$, where $\tau$ is the Fourier transform of the IRF, $\phi_{\mathrm{o}}$ is the power spectrum of the 'object' or true function (approximated by that of the raw data) and $\phi_{\mathrm{n}}$ is the power spectrum of the noise, which, assuming white noise, can be found easily from the Fourier transform of the raw data.

Received 11 July 2008; accepted 19 December 2008; published online 25 January 2009

\section{References}

1. Kurtsiefer, C., Mayer, S., Zarda, P. \& Weinfurter, H. Stable solid-state source of single photons. Phys. Rev. Lett. 85, 290-293 (2000).

2. Michler, P. et al. Quantum correlation among photons from a single quantum dot at room temperature. Nature 406, 968-970 (2000). 
3. Kiraz, A., Atature, M. \& Imamoglu, A. Quantum-dot single-photon sources: Prospects for applications in linear optics quantum-information processing. Phys. Rev. A 69, 032305 (2004).

4. Xu, X. D. et al. Coherent optical spectroscopy of a strongly driven quantum dot. Science 317, 929-932 (2007).

5. Wrigge, G. et al. Efficient coupling of photons to a single molecule and the observation of its resonance fluorescence. Nature Phys. 4, 60-66 (2008).

6. Basché, T., Moerner, W. E., Orrit, M. \& Talon, H. Photon antibunching in the fluorescence of a single dye molecule trapped in a solid. Phys. Rev. Lett. 69, 1516-1519 (1992).

7. Batalov, A. et al. Temporal coherence of photons emitted by single nitrogen-vacancy defect centers in diamond using optical Rabi-oscillations. Phys. Rev. Lett. 100, 077401 (2008).

8. Santori, C. et al. Indistinguishable photons from a single-photon device. Nature 419, 594-597 (2002).

9. Bennett, C. H. \& Brassard, G. Proc. IEEE Int. Conf. Computers, Systems and Signal Processing, December 1984, Bangalore, India 175-179.

10. Peres, A. Separability criterion for density matrices. Phys. Rev. Lett. 77, 1413-1415 (1996).

11. Fattal, D. et al. Entanglement formation and violation of Bell's inequality with a semiconductor single photon source. Phys. Rev. Lett. 92, 037903 (2004).

12. Stevenson, R. M. et al. A semiconductor source of triggered entangled photon pairs. Nature 439, 179-182 (2006).

13. Kamada, H. et al. Exciton Rabi oscillation in a single quantum dot. Phys. Rev. Lett. 87, 246401 (2001).

14. Stievater, T. H. et al. Rabi oscillations of excitons in single quantum dots. Phys. Rev. Lett. 87, 133603 (2001).

15. Htoon, H. et al. Interplay of Rabi oscillations and quantum interference in semiconductor quantum dots. Phys. Rev. Lett. 88, 087401 (2002).

16. Zrenner, A. et al. Coherent properties of a two-level system based on a quantum-dot photodiode. Nature 418, 612-614 (2002).

17. $\mathrm{Wu}, \mathrm{F}$. Y., Grove, R. E. \& Ezekiel, S. Investigation of the spectrum of resonance fluorescence induced by a monochromatic field. Phys. Rev. Lett. 35 1426-1429 (1975).

18. Kimble, H. J., Dagenais, M. \& Mandel, L. Photon antibunching in resonance fluorescence. Phys. Rev. Lett. 39, 691-695 (1977).

19. Muller, A. et al. Resonance fluorescence from a coherently driven semiconductor quantum dot in a cavity. Phys. Rev. Lett. 99, 187402 (2007).
20. Mollow, B. R. Power spectrum of light scattered by two-level systems. Phys. Rev. 188, 1969-1975 (1969).

21. Cohen-Tannoudji, C., Dupont-Roc, J. \& Grynberg, G. Atom-Photon Interactions (Wiley, 1992).

22. Bayer, M. et al. Inhibition and enhancement of the spontaneous emission of quantum dots in structured microresonators. Phys. Rev. Lett. 86, 3168-3171 (2001)

23. Solomon, G. S., Pelton, M. \& Yamamoto, Y. Single-mode spontaneous emission from a single quantum dot in a three-dimensional microcavity. Phys. Rev. Lett. 86, 3903-3906 (2001).

24. Scully, M. O. \& Zubairy, M. S. Quantum Optics (Cambridge Univ. Press, 1997).

25. Högele, A. et al. Voltage-controlled optics of a quantum dot. Phys. Rev. Lett. 93, 217401 (2004).

26. Jundt, G. et al. Observation of dressed excitonic states in a single quantum dot. Phys. Rev. Lett. 100, 177401-177404 (2008).

27. Torrey, H. C. Transient nutations in nuclear magnetic resonance. Phys. Rev. 76, 1059-1068 (1949).

28. Michler, P. et al. A quantum dot single-photon turnstile device. Science 290, 2282-2285 (2000).

29. Wang, X. Y. et al. Photoluminescence intermittency of InGaAs/GaAs quantum dots confined in a planar microcavity. Nano Lett. 5, 1873-1877 (2005).

30. Jansson, P. A. (ed.) Deconvolution of Images and Spectra 2 nd edn (Academic, 1997).

\section{Acknowledgements}

We gratefully acknowledge funding from the National Science Foundation NSF-FRG grant number DMR-0606485, NSF-IGERT grant number DGE-0549417 and the Texas Advanced Research Program, and thank W. Yao for fruitful discussions.

\section{Author contributions}

W.M., M.X. and G.J.S. fabricated the sample. E.B.F., A.M., D.G.D. and C.K.S. conceived the experiments. E.B.F, A.M., J.W.R. and S.F. carried out the experimental work. E.B.F. and C.K.S. analysed the data. E.B.F., A.M. and C.K.S. wrote the article, with input from the other co-authors.

\section{Additional information}

Supplementary Information accompanies this paper on www.nature.com/naturephysics. Reprints and permissions information is available online at http://npg.nature.com/ reprintsandpermissions. Correspondence and requests for materials should be addressed to E.B.F. or C.K.S 